\title{
Reply to Letter to the Editor re: "Radiologically guided placements of totally implantable central venous port: observations about right internal jugular access"
}

\author{
Ulf Karl-Martin Teichgräber
}

Received: 17 March 2011 / Accepted: 28 April 2011 /Published online: 22 May 2011

(C) European Society of Radiology 2011

Dear Editor,

We thank the correspondents for their comments. Our low periprocedural complication rate is related to our very standardized implantation technique. We start with an ultrasound examination before the implantation to verify the appropriate venous access and to rule out venous thrombosis. All central venous punctures were performed under ultrasound guidance and the guide wire and catheter placement were fluoroscopically controlled. Our early and late complication rate did not differ from other studies. A final chest X-ray should be always performed to exclude a pneumothorax or a large mediastinal haematoma. The final results were always documented by $\mathrm{x}$-ray in all our patients.
For the Seldinger technique we used a $18 \mathrm{G}$-singlewall entry needle for all port implantations. A micropuncture needle set is recommended for residents starting with the ultrasound guided puncture technique of the internal jugular vein (IJV) or for partially thrombosed central veins. The IJV typically presents itself with a relatively large lumen. In that case the success rate is very high in the hands of an experienced interventional radiologist.

Yours sincerely,

Ulf Teichgraeber on behalf of the authors.

U. K.-M. Teichgräber $(\bowtie)$

Institut für Diagnostische und Interventionelle Radiologie,

Charité Universitätsmedizin Berlin,

Charitéplatz 1,

10117 Berlin, Germany

e-mail: ulf.teichgraeber@charite.de 